\title{
Funcionalidades de la gamificación: Classcraft y Moodle en el marco de Octalysis
}

Sánchez-Pacheco, Carlos Luis

Sociedad de Investigación Pedagógica Innovar, Ecuador

carlossanchez21@hotmail.com

Resumen - La gamificación es una técnica reciente que consiste en aplicar elementos del juego en contextos ajenos al juego. Existen marcos teóricos que sugieren un modelo para aplicar estos elementos en la implementación de soluciones concretas. En este artículo se analiza dos sistemas de gestión de aprendizaje, Classcraft y Moodle, bajo la lente del marco de Octalysis. A pesar de que las dos plataformas proporcionan varios elementos del juego, que combinados entre sí buscan aumentar la motivación de los estudiantes.

Palabras clave - Classcraft; Gamificación; Moodle; Octalysis; Sistemas de gestión de aprendizaje;

Abstract - Gamification is a recent technique that involves applying elements of the game in contexts outside the game. There are theoretical frameworks that suggest a model to apply these elements in the implementation of concrete solutions. This article discusses two learning management systems, Classcraft and Moodle, under the lens of the Octalysis framework. Even though the two platforms provide various elements of the game, which combined with each other seek to increase the motivation of the students.

Keywords - Classcraft; Gamification; Moodle; Octalysis; Learning management systems;

Interconectando Saberes, 2020

ISSN: 2448-8704

\section{INTRODUCCIÓN}

El término "gamificación" es reciente, un neologismo derivado del término anglófono "gamification", con una traducción alternativa de "ludificación", más en latín, pero menos utilizado. Para (Deterding, 2012) "Gamificación" es el uso de un elemento de diseño de videojuegos en contextos que no son juegos. Otros autores defienden perspectivas complementarias. Por ejemplo, (Zichermann \& Cunningham, 2011) refieren que la gamificación es un proceso de pensamiento de juego, es decir, pensamiento basado en la estructura y dinámica de los juegos, basado en la mecánica de los juegos, con el objetivo de involucrar a los usuarios en la resolución de problemas.

Siguiendo la misma línea de pensamiento, (Kapp, 2012) sugiere que el pensamiento del juego es probablemente el elemento más importante de la gamificación, responsable de convertir una actividad cotidiana en una actividad que agregue

Fecha de Recepción: 14 de marzo de 2020

Fecha de Aceptación: 08 de mayo de 2020 Fecha de Publicación: 24 de julio de 2020 
elementos de competencia, cooperación y narrativa. Por lo tanto, la gamificación se centra en la motivación humana, por lo que es posible considerarla un tipo de "concepción centrada en el ser humano", como defiende (Chou, 2016), sin olvidar que los elementos del juego no se limitan a los aspectos motivacionales. Este proceso de concepción enfatiza los elementos sociológicos, antropológicos y psicológicos del ser humano, que se originan en emociones, sentimientos, inseguridades, motivación y razones para que los usuarios se involucren en ciertas tareas 0 actividades.

Debido al creciente interés que el concepto de gamificación ha causado en algunos sectores, incluida la educación (Barata, Gama, \& Gonçalves, 2013; Klock, de Carvalho, Eduardo Rosa, \& Gasparini, 2015; Dichev \& Dicheva, 2017), han surgido modelos o marcos teóricos para guiar el diseño y la evaluación de actividades gamificadas. El objetivo de este trabajo es utilizar el marco de Octalysis para verificar qué principios funcionales de gamificación están presentes en dos sistemas de gestión del aprendizaje que pueden usarse en la práctica pedagógica.

\section{Metodología}

Para la elaboración de este trabajo, se analizaron dos entornos virtuales de aprendizaje, la plataforma Classcraft, disponible en https://www.classcraft.com/y la plataforma Moodle, disponible en: https://moodle.org/, ambos utilizados por primer autor en su práctica docente. Se elaboró una cuadrícula de análisis (tabla 1) con una descripción detallada de todos los elementos del juego presentes en cada eje del modelo de Octalysis, luego se analizaron las dos plataformas en función de esta cuadrícula.

Cabe señalar que hay varios suplementos de gamificación para expandir el potencial de Moodle a este nivel (consulte https://moodle.org/plugins/browse.php?list=set\&id =88na), pero el análisis que hacemos aquí se centra en características estándar disponibles en todas las instalaciones de Moodle.

\section{Resultados}

\section{Presentación del Marco de Octalysis}

El nombre Octalysis proviene de la forma geométrica (octogonal) utilizada por su creador (Chou, 2016) para representar los ocho ejes de análisis del marco. Cada eje representa una dimensión diferente, apoyando la participación del jugador en actividades gamificadas: significado (significado y vocación épicos), empoderamiento de la creatividad (empoderamiento de la creatividad y la retroalimentación), influencia social (influencia social y relación), imprevisibilidad (imprevisibilidad y curiosidad), contención (pérdida y evitación), escasez (escasez e impaciencia), posesión (propiedad y posesión) y logro (desarrollo y realización), con los aspectos positivos (Sombrero blanco) en la parte superior y los aspectos negativos en la parte inferior (Sombrero negro); a la derecha los factores de motivación extrínseca, a la izquierda los factores de motivación intrínseca. 
Figura 1. Marco de Octalysis

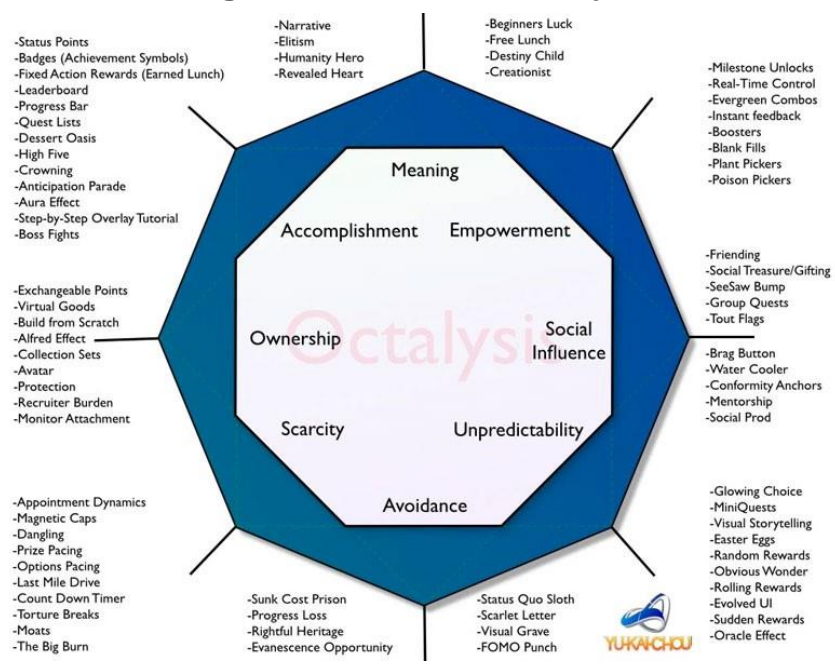

Fuente:

https://repositorioaberto.uab.pt/bitstream/10400.2/7294/1/Ferr eira\%20et\%20al.pdf

\section{1) Significado}

Este eje analiza si la experiencia tiene un significado para el usuario, lo hace sentir que será parte de algo importante para él y para los demás. Por ejemplo, cubre aspectos como la narrativa, que proporciona un contexto para la actividad, una razón para tener que llevar a cabo la acción, quién debe llevarla a cabo, cuáles son los objetivos que se deben lograr, además de dar sentido a los métodos y las limitaciones de su ejecución.

La plataforma Classcraft tiene un módulo, llamado "misiones" que coloca al estudiante en la narrativa y lo guía en las actividades que se llevarán a cabo hasta completar la misión. Cada ítem representa una actividad con 4 aspectos: la historia, que asegura la continuidad de la narración; la tarea, que proporciona información sobre la actividad a desarrollar; la metodología de ejecución; y el resultado esperado. La narración cuenta una historia a lo largo de toda la misión y se coloca al estudiante en el centro de la misma, y se espera que realice ciertas acciones que solo dependen de él. El alumno, al completar una actividad, recibe otra. La secuencia le dará continuidad narrativa hasta el final de la misión, haciendo que el estudiante sea el centro de la narración, para que se sienta parte de algo más grande.

En la plataforma Moodle no encontramos ninguna referencia explícita a este eje, sin embargo, la estructura de las unidades curriculares es secuencial y se divide en temas, que pueden integrar libremente elementos textuales $y$ multimedia, aspectos que pueden usarse para transmitir una narrativa a lo largo del camino de aprendizaje, sin embargo, sin poder individualizar esta secuencia para cada alumno.

\section{2) Realización}

La existencia de recompensas tiene como objetivo mantener a los estudiantes involucrados en la actividad y hacer esfuerzos para mejorar, a fin de completar con éxito los desafíos propuestos. Superar problemas, cambiar comportamientos y desarrollar habilidades o competencias pueden ser ejemplos de desafíos.

En la plataforma Classcraft, las recompensas se otorgan en forma de diferentes tipos de puntos. Los $\mathrm{XP}$ son puntos de experiencia que el estudiante gana cada vez que finaliza con éxito una tarea; Las monedas de oro GP le permiten comprar equipos y mascotas en la economía del juego; Los AP son para que el estudiante adquiera poderes; Los 
puntos de salud HP, representan la capacidad de daño que el estudiante puede acumular si presenta comportamientos incorrectos. Si pierde todo HP, el estudiante "cae en la batalla" en el contexto narrativo de Classcraft y tendrá una consecuencia.

En la plataforma Moodle, las recompensas se otorgan en forma de insignias que el estudiante recoge. Estas son medallas virtuales, que se pueden otorgar automáticamente, después de que el alumno complete una serie de actividades, o de forma manual, con la posibilidad de que el maestro otorgue la medalla, por ejemplo, la medalla "Alumno más participante".

\section{3) Empoderamiento de la creatividad.}

El empoderamiento de la creatividad se refiere a la participación de los usuarios en procesos en los que se desarrolla la capacidad de inventar estrategias para lograr un fin particular.

En la plataforma Classcraft, el estudiante al desarrollar una misión se enfrenta a varios caminos posibles, cada uno de ellos con actividades que deben llevarse a cabo. Cada actividad implica desarrollar un conjunto de tareas con las herramientas más apropiadas. Si un estudiante no alcanza la meta deseada, siempre puede regresar y seguir otro camino y elegir otras herramientas, esta elección de herramientas requiere la capacidad de definir su estrategia para resolver los problemas propuestos.
En la plataforma Moodle, la actividad "Prueba" está disponible. Cuando un estudiante responde a una prueba, tiene comentarios inmediatos sobre su respuesta. Si el maestro configura la prueba para permitir varios intentos, el estudiante puede volver a responder (con o sin penalización), pero antes de eso, Moodle lo llevará a una página con información detallada sobre el contenido que estaba mal. De esta manera, existe la posibilidad de volver a intentarlo, pero esta dinámica solo implica nuevas estrategias si las preguntas mismas están diseñadas para no tener una respuesta obvia. Por ejemplo: las preguntas centradas en el conocimiento objetivo solo analizan su dominio por parte de los estudiantes. Las preguntas que permiten, a través de intentos sucesivos, descubrir pistas sobre la identidad de un objeto, como si se tratara un juego de detectives, ya permiten el desarrollo de estrategias alternativas para cada intento.

\section{4) Propiedad}

Este eje abarca funcionalidades que permiten al alumno ser el titular de algo. Puede ser equipo $u$ otros recursos, como utensilios o mascotas.

En la plataforma Classcraft, el estudiante, al acumular puntos, puede comprar equipo para personalizar el avatar que lo representa en la plataforma, además de mascotas virtuales y entrenarlos. Hay varios tipos de equipos y mascotas que el estudiante puede elegir y personalizar, y lo hace de acuerdo con su gusto personal. 
En la plataforma Moodle, no encontramos evidencia de este eje.

\section{5) Influencia social}

Este eje se refiere a actividades que involucran a otras personas, sus gustos y opiniones en relación con ciertos temas (el marco menciona la tutoría, la aceptación, las reacciones sociales, el compañerismo, la competencia e incluso la envidia como ejemplos). Este eje, por ejemplo, incluye la tutoría y el intercambio de conocimientos entre estudiantes experimentados y neófitos.

En la plataforma Classcraft, los estudiantes trabajan en equipo, todos son responsables del comportamiento de todos los miembros del equipo, si los comportamientos son apropiados con las reglas previamente aceptadas, benefician a todos, de lo contrario son penalizados. Esta estructura es naturalmente propicia para las actividades en esta área.

La plataforma Moodle proporciona varias características a este nivel. Es posible crear un bloque de resultados, que muestre los mejores resultados obtenidos por los estudiantes, o grupos de estudiantes, en una actividad dada. La configuración de la agenda para mostrar la posición relativa de cada estudiante, en relación con la posición de sus compañeros, es similar.
Obviamente, la perspectiva participativa de muchas de las actividades predefinidas de Moodle está orientada hacia este aspecto. Por ejemplo, es posible estructurar a los estudiantes en grupos, estrictamente 0 asignándolos a diferentes actividades; establecer foros de debate con fines y dinámicas sociales; Proponer actividades de producción de contenido cooperativo, a través de glosarios y wikis. O una interacción aún más intensa a través del Taller, donde los estudiantes alternan roles entre la producción de contribuciones y la evaluación con comentarios de las contribuciones de colegas.

\section{6) Escasez}

El eje de escasez defiende que ciertos bienes 0 servicios deben ser raros o de acceso limitado. Pueden ser elementos virtuales, físicos, limitaciones de tiempo, libertades de acción o cualquier otro aspecto.

En la plataforma Classcraft, la compra de mascotas y ciertos equipos depende de la cantidad de monedas de oro que tenga el estudiante, y gastarlas en una no es posible gastar en otras. Algunos de estos recursos solo están disponibles después de alcanzar un cierto nivel, es decir, se ven con menos frecuencia ya que no todos los participantes pueden acceder a ellos. En (figura 2) presentamos un avatar personalizado con una mascota por empresa y ya entrenado. 
Figura 2. Personalización de Avatar y Mascotas (Classcraft)

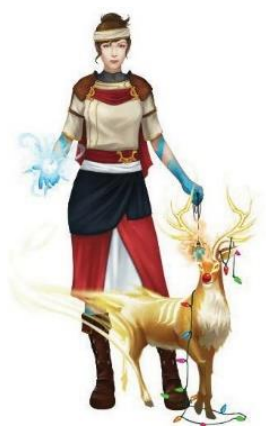

Fuente:

https://repositorioaberto.uab.pt/bitstream/10400.2/7294/1/Ferr eira\%20et\%20al.pdf

En la plataforma Moodle, no encontramos referencias a este eje, pero es concebible que un maestro pueda explicar las limitaciones en la configuración o las reglas de ciertas actividades. Por ejemplo, hay un foro o un conjunto de herramientas a las que solo pueden acceder los primeros participantes, una prueba con pistas que solo está disponible en ciertos momentos del día u otros enfoques similares

\section{7) Imprevisibilidad}

El eje de imprevisibilidad recomienda que sea necesario continuar las actividades para descubrir qué sucederá, es decir, que algo que el estudiante no puede esperar puede suceder.

En la plataforma Classcraft, esta técnica se utiliza en las misiones: cuando el estudiante termina una actividad, la siguiente se hace visible, así como una parte del mapa, cuando el mapa se vuelve completamente visible es porque la misión ha terminado, pero mientras se está ejecutando no es predecible cuál será su desarrollo. El módulo de eventos aleatorios es otra característica que apunta a la imprevisibilidad: al ser solo frases simples que presentan una condición especial durante la clase, conducen al hecho de que solo los participantes en la clase pueden ver lo que sucede.

En la plataforma Moodle, no encontramos evidencia de este eje, pero cómo es posible ocultar y mostrar temas o actividades y recursos, el maestro puede controlar la visibilidad de estos componentes para dinamizar este eje.

\section{8) Contención}

Este eje está dedicado a situaciones en las que el alumno intenta evitar consecuencias negativas. Por ejemplo, saber que, si una tarea no se realiza dentro de un tiempo determinado, se le penalizará o no podrá completarla.

En la plataforma Classcraft, una de las características a lo largo de este eje son los temporizadores de conteo, progresivos (Figura 3) y regresivos, que le muestran al estudiante los minutos para pasar mientras ejecuta una tarea que puede estar respondiendo una prueba ("prueba"), resuelva un rompecabezas, lea un texto, el tiempo transcurrido se refleja en la pérdida del valor máximo de recompensa en puntos de experiencia. El mismo tipo de funcionalidad está presente en todas las situaciones que pueden conducir a la pérdida de puntos de salud (HP). 
Figura 3. Temporizador ascendente (Classcraft)

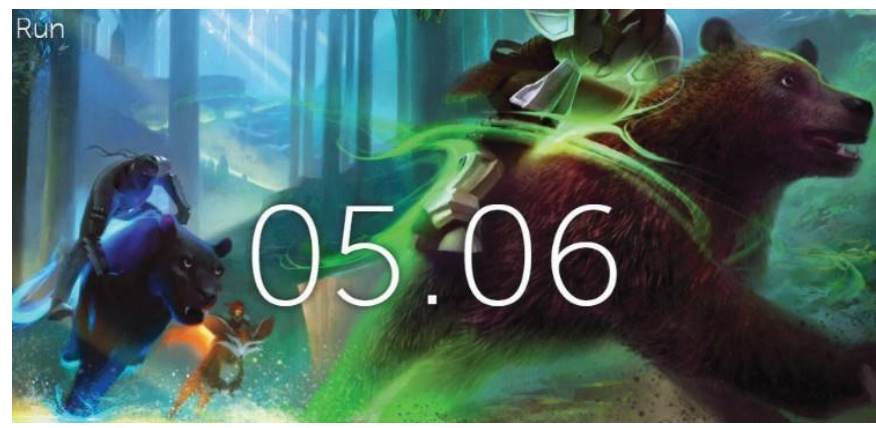

Fuente:

https://repositorioaberto.uab.pt/bitstream/10400.2/7294/1/Ferr eira\%20et\%20al.pdf

En la plataforma Moodle, las configuraciones limitantes para la participación están potenciando este eje. Por ejemplo, límites de tiempo para enviar trabajos o límites para intentar responder preguntas o enviar trabajos (Figura 4).

Figura 4. Prueba con límite de tiempo de respuesta (Moodle)

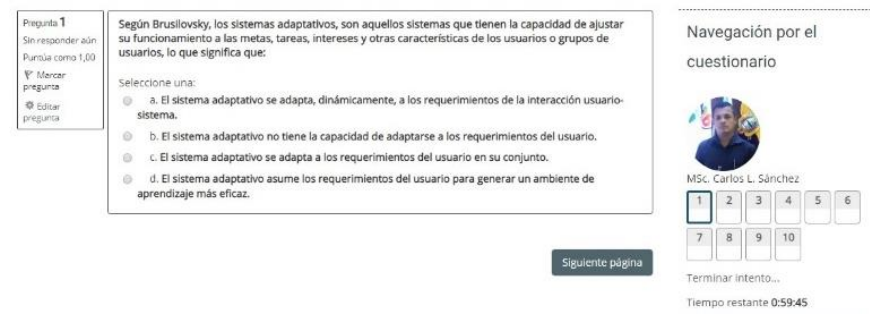

Fuente: Elaboración propia.

\section{HERRAMIENTA DE OCTALYSIS APLICADA A AMBOS ENTORNOS DE APRENDIZAJE.}

En (figura 5), presentamos gráficamente la mecánica presente en la plataforma Moodle de acuerdo con el modelo de Octalysis.
Figura 5. Herramienta de Octalysis aplicada a la plataforma Moodle.

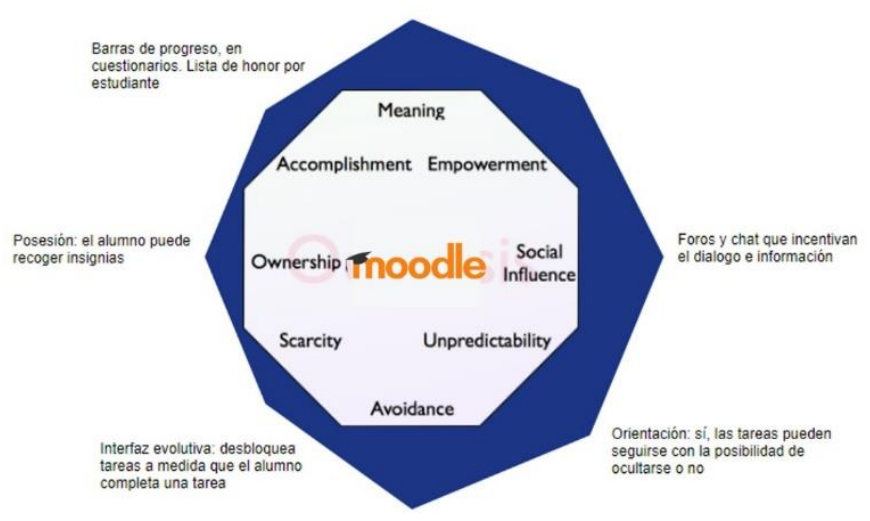

Fuente:

https://repositorioaberto.uab.pt/bitstream/10400.2/7294/1/Ferr eira\%20et\%20al.pdf

En (figura 6), presentamos gráficamente la mecánica presente en la plataforma Classcraft de acuerdo con el modelo de Octalysis.

Figura 6. Herramienta de Octalysis aplicada a la plataforma Classcraft

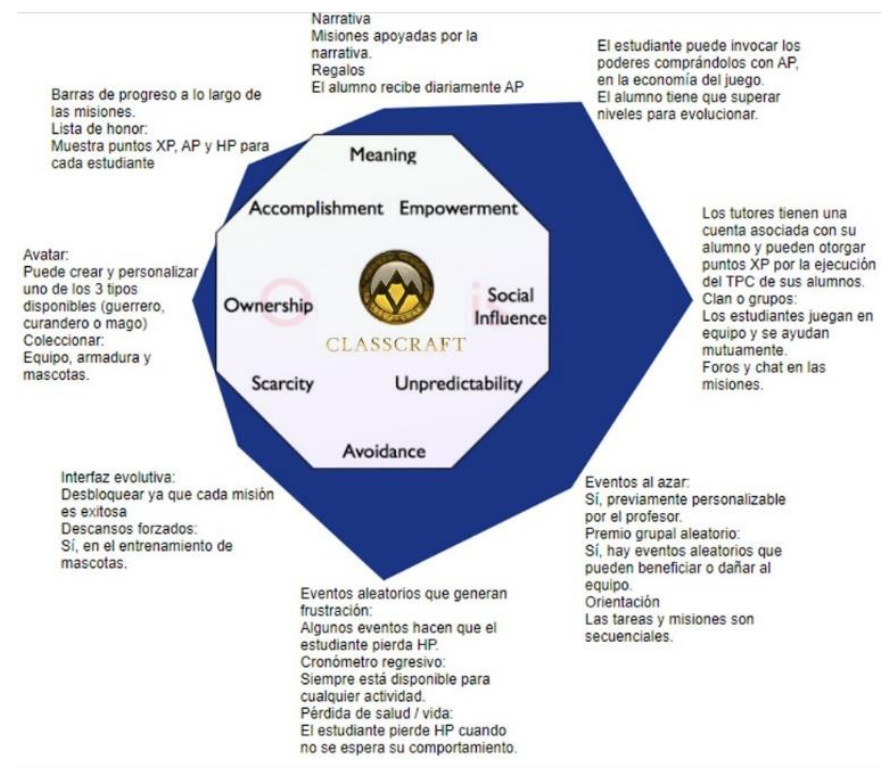

Fuente:

https://repositorioaberto.uab.pt/bitstream/10400.2/7294/1/Ferr eira\%20et\%20al.pdf

Relevancia del estudio 
El presente estudio evalúa las plataformas de gamificación más utilizadas en la educación no superior. En este estudio se analizaron las funcionalidades objetivas de cada plataforma, Classcraft y Moodle, así como algunas de las funcionalidades que puede implementar el profesor. En (tabla 1), se expone brevemente la mecánica presente en las dos plataformas bajo análisis.

Tabla 1. Mecánica presente en las plataformas Classcraft y Moodle

\begin{tabular}{|c|c|c|c|}
\hline Ejes & Mecánicas & Classcraft & Moodle \\
\hline Significado & $\begin{array}{l}\text { Narrativa } \\
\text { Regalos }\end{array}$ & $\begin{array}{l}\text { Misiones apoyadas por la narrativa. } \\
\text { El alumno recibe diariamente AP }\end{array}$ & $\begin{array}{l}\text { No } \\
\text { No }\end{array}$ \\
\hline \multirow[t]{3}{*}{ Realización } & Barras de progreso & Sí, en misiones & Sí, personalizables \\
\hline & Tablas de honor & $\begin{array}{l}\text { Sí. Tabla de puntos (AP, } \\
\text { XP y HP) de cada alumno }\end{array}$ & $\mathrm{Si}$ \\
\hline & Experimenta puntos & $\begin{array}{l}\text { Sí, cada vez que el alumno realiza una } \\
\text { actividad o demuestra un } \\
\text { comportamiento apropiado }\end{array}$ & $\mathrm{Si}$ \\
\hline
\end{tabular}

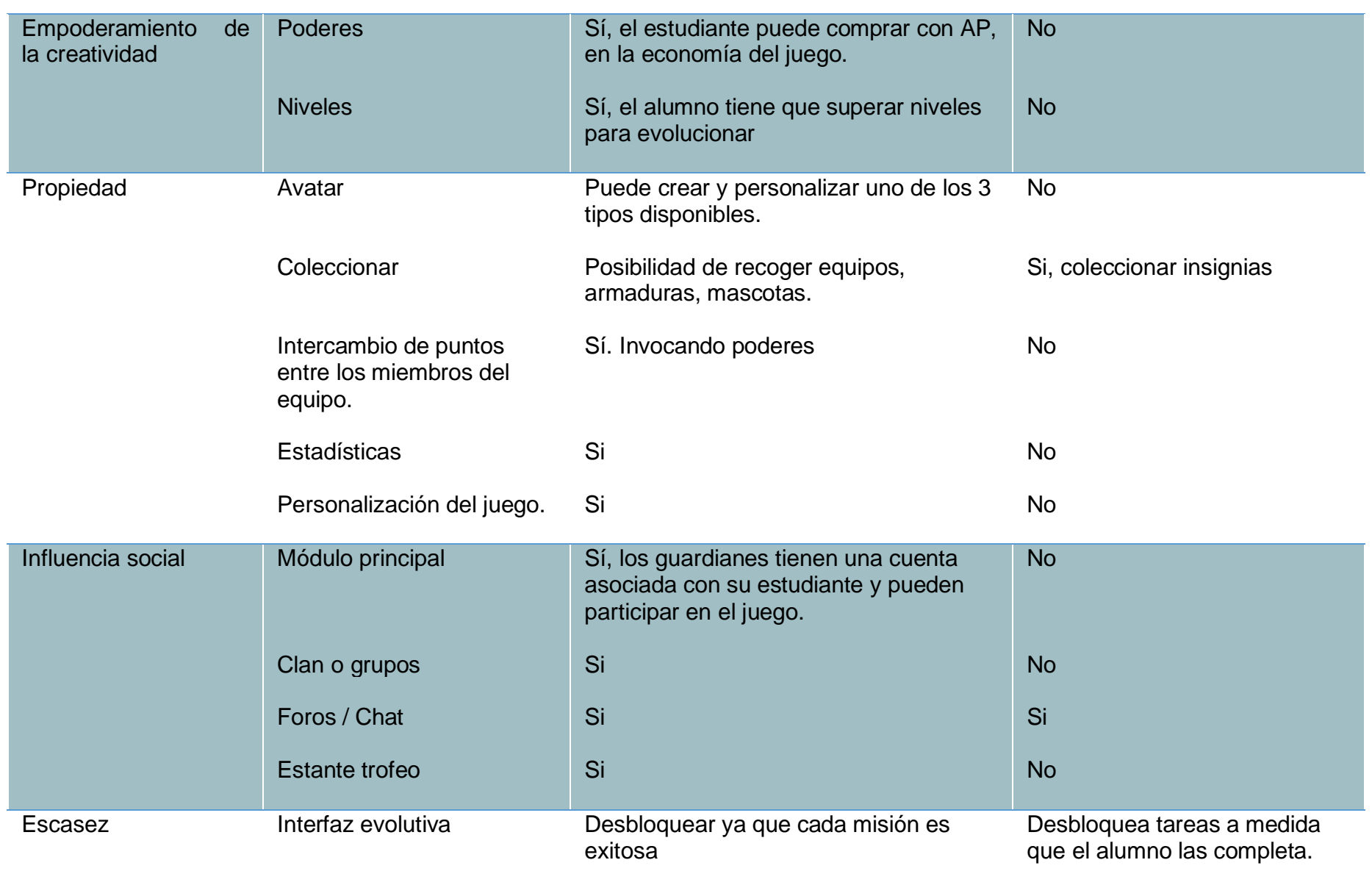

Descansos forzados

Sí, al entrenar mascotas.

No 


\begin{tabular}{|c|c|c|c|}
\hline Ejes & Mecánicas & Classcraft & Moodle \\
\hline \multirow[t]{3}{*}{ Imprevisibilidad } & Eventos al azar & $\begin{array}{l}\text { Sí, previamente personalizable por el } \\
\text { profesor. }\end{array}$ & No \\
\hline & $\begin{array}{l}\text { Premios grupales } \\
\text { aleatorios }\end{array}$ & Sí. Eventos aleatorios & No \\
\hline & Orientación & Sí, las tareas son secuenciales. & $\begin{array}{l}\text { Sí, las tareas se pueden } \\
\text { secuenciar y la tarea se puede } \\
\text { ocultar o no. }\end{array}$ \\
\hline \multirow[t]{2}{*}{ Contención } & $\begin{array}{l}\text { Eventos aleatorios que } \\
\text { generan frustración. } \\
\text { Cronómetros }\end{array}$ & \multicolumn{2}{|l|}{$\begin{array}{l}\text { Sí, algunos eventos hacen que el } \\
\text { estudiante pierda HP } \\
\text { Sí. Siempre disponible para cualquier } \\
\text { actividad. }\end{array}$} \\
\hline & Pérdida de salud / vida & \multicolumn{2}{|l|}{$\begin{array}{l}\text { Sí, el estudiante pierde HP cuando su } \\
\text { comportamiento no es positivo. }\end{array}$} \\
\hline
\end{tabular}

Fuente: Elaboración propia.

\section{CONCLUSIONES}

En este artículo analizamos las funcionalidades de gamificación disponibles en los entornos de aprendizaje de Classcraft y Moodle, utilizando el marco de Octalysis como referencia. Descubrimos que la plataforma Classcraft tiene al menos una función de gamificación para cada eje del modelo Octalysis. La plataforma Moodle también presenta al menos una funcionalidad de gamificación en la mayoría de los ejes, pero en muchos de ellos esta funcionalidad no se presenta como tal, y su uso en este sentido depende de estrategias superpuestas por el profesor, a través de reglas textuales o adaptación de configuraciones.

Cabe señalar que la plataforma Moodle no se desarrolló específicamente para contener elementos del juego, sin embargo, su versatilidad permite el tipo de enfoques que se describen aquí. También hay suplementos específicos para darle otras características, que no se analizaron en este trabajo. La plataforma Classcraft fue pensada y diseñada para este propósito, por lo que no es sorprendente que tenga características cada vez más diversas. Sin embargo, esta comparación nos permite aclarar la percepción de cuáles son las diferencias cualitativas efectivas entre una plataforma de gamificación especializada y una adaptación de una plataforma genérica.

La adaptación de plataformas en la gamificación requiere de un proceso de análisis exhaustivo debido a las cargas de motivación que se le imprime a una plataforma, esto depende de la capacidad de aprendizaje de los estudiantes; esto puede ser el punto de partida para posibles trabajos futuros en los cuales se analice esa capacidad y se pueda determinar con claridad los elementos que deberían estar inmersos.

\section{REFERENCIAS}

Barata, G., Gama, S., \& Gonçalves, D. (2013). Melhorando o Ensino universitário com a Gamificação.

Borges, S., Durelli, V., Reis, H., \& Isotani, S. (2014). A Systematic Mapping on Gamification Applied to Education. doi:10.1145/2554850.2554956 
Burke, B. (2015). Gamify Cómo la gamificación motiva a las personas a hacer cosas extraordinarias. DVS Editora.

Chou, Y. (2016). Actionable Gamification: beyond points, badges and leaderboards. Revista Internacional de Organizaciones, 137-144. doi:10.17345/rio18

Deterding, S. (2012). Gamification: designing for motivation. Interactions, New York, v. 19, 1417.

Dichev, C., \& Dicheva, D. (2017). Gamifying education: what is known, what is believed and what remains uncertain: a critical review. doi:10.1186/s41239-017-0042-5

Kapp, K. M. (2012). The gamification of learning and instruction: game-based methods and strategies for training and education. San Francisco, CA: Pfeiffer.

Klock, A., de Carvalho, M., Eduardo Rosa, B., \& Gasparini, I. (2015). Análisis de técnicas de gamificación en entornos virtuales de aprendizaje. ENOTE - Revista Novas Tecnologias na Educação. 12, 1-10. doi:https://doi.org/10.22456/16791916.53496

Zichermann, G., \& Cunningham, C. (2011). Gamification by Design. Canada: O'Reilly Media. 